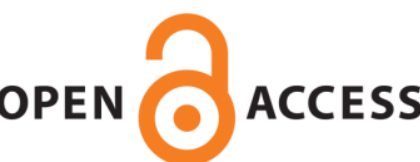

UWS Academic Portal

\title{
Coatings and surface treatments for enhanced performance suspensions for future gravitational wave detectors
}

Birney, Ross; Cumming, Alan Vernal ; Campsie, Paul; Gibson, Desmond; Hammond, Giles D. ; Hough, Jim ; Martin, lain W.; Reid, Stuart; Rowan, Sheila; Song, Shigeng; Talbot, Curtis;

Vine, David; Wallace, Gavin

Published in:

Classical and Quantum Gravity

DOI:

10.1088/1361-6382/aa9354

E-pub ahead of print: 15/11/2017

Document Version

Peer reviewed version

Link to publication on the UWS Academic Portal

Citation for published version (APA):

Birney, R., Cumming, A. V., Campsie, P., Gibson, D., Hammond, G. D., Hough, J., Martin, I. W., Reid, S.,

Rowan, S., Song, S., Talbot, C., Vine, D., \& Wallace, G. (2017). Coatings and surface treatments for enhanced performance suspensions for future gravitational wave detectors. Classical and Quantum Gravity, 34, [235012]. https://doi.org/10.1088/1361-6382/aa9354

\section{General rights}

Copyright and moral rights for the publications made accessible in the UWS Academic Portal are retained by the authors and/or other copyright owners and it is a condition of accessing publications that users recognise and abide by the legal requirements associated with these rights. 
ACCEPTED MANUSCRIPT

\section{Coatings and surface treatments for enhanced performance suspensions for future gravitational wave detectors}

To cite this article before publication: Ross Birney et al 2017 Class. Quantum Grav. in press https://doi.org/10.1088/1361-6382/aa9354

\section{Manuscript version: Accepted Manuscript}

Accepted Manuscript is "the version of the article accepted for publication including all changes made as a result of the peer review process, and which may also include the addition to the article by IOP Publishing of a header, an article ID, a cover sheet and/or an 'Accepted Manuscript' watermark, but excluding any other editing, typesetting or other changes made by IOP Publishing and/or its licensors"

This Accepted Manuscript is @ 2017 IOP Publishing Ltd.

During the embargo period (the 12 month period from the publication of the Version of Record of this article), the Accepted Manuscript is fully protected by copyright and cannot be reused or reposted elsewhere.

As the Version of Record of this article is going to be / has been published on a subscription basis, this Accepted Manuscript is available for reuse under a CC BY-NC-ND 3.0 licence after the 12 month embargo period.

After the embargo period, everyone is permitted to use copy and redistribute this article for non-commercial purposes only, provided that they adhere to all the terms of the licence https://creativecommons.org/licences/by-nc-nd/3.0

Although reasonable endeavours have been taken to obtain all necessary permissions from third parties to include their copyrighted content within this article, their full citation and copyright line may not be present in this Accepted Manuscript version. Before using any content from this article, please refer to the Version of Record on IOPscience once published for full citation and copyright details, as permissions will likely be required. All third party content is fully copyright protected, unless specifically stated otherwise in the figure caption in the Version of Record.

View the article online for updates and enhancements. 


\title{
Coatings and surface treatments for enhanced performance suspensions for future gravitational wave detectors
}

\author{
R Birney ${ }^{1,2} \dagger$, A V Cumming ${ }^{3}$, P Campsie $^{1,2}$, D Gibson ${ }^{1}$, G \\ D Hammond $^{3}$, J Hough ${ }^{3}$, I W Martin ${ }^{3}$, S Reid ${ }^{1,2 \dagger}$, S \\ Rowan $^{3}$, S Song ${ }^{1}$, C Talbot ${ }^{1}$, D Vine ${ }^{1}$, G Wallace ${ }^{1}$ \\ ${ }^{1}$ SUPA $\ddagger$ Institute of Thin Films, Sensors and Imaging, School of Engineering and \\ Computing, University of the West of Scotland, Paisley PA1 2BE, UK \\ ${ }^{2}$ SUPA $\ddagger$ Department of Biomedical Engineering, University of Strathclyde, \\ Glasgow G4 0NW, UK \\ ${ }^{3}$ SUPA Institute for Gravitational Research, School of Physics and Astronomy, \\ University of Glasgow, Glasgow, G12 8QQ, UK \\ E-mail: Ross.Birney@strath.ac.uk, Stuart.Reid@strath.ac.uk
}

\begin{abstract}
Further improvements in the low frequency sensitivity of gravitational wave detectors are important for increasing the observable population of astrophysical sources, such as intermediate mass compact black hole binary systems. Improvements in the lower stagemirror and suspension systems will set challenging targets for the required thermal noise performance of the cantilever blade springs, which provide vertical softness and, thus, isolation to the mirror suspension stack. This is required due to the coupling between the vertical and horizontal axes due to the curvature of the Earth. This can be achieved through use of high mechanical Q materials, which are compatible with cryogenic cooling, such as crystalline silicon. However, such materials are brittle, posing further challenges for assembly/jointing and, more generally, for long-term robustness. Here, we report on experimental studies of the breaking strength of silicon at room temperature, via both tensile and 4-point flexural testing; and on the effects of various surface treatments and coatings on durability and strength. Single- and multi-layer DLC (diamond-like carbon) coatings, together with magnetron-sputtered silica and thermally-grown silica, are investigated, as are the effects of substrate preparation and argon plasma pre-treatment. Application of single- or multi-layer DLC coatings can significantly
\end{abstract}

$\dagger$ corresponding author

$\ddagger$ Scottish Universities Physics Alliance 
improve the failure stress of silicon flexures, in addition to improved robustness for handling (assessed through abrasion tests). Improvements of up to $80 \%$ in tensile strength, a twofold increase in flexural strength, in addition to a 6.4 times reduction in the vertical thermal noise contribution of the suspension stack at 10 $\mathrm{Hz}$ are reported (compared to current Advanced LIGO design). The use of silicon blade springs would also significantly reduce potential "crackling noise" associated with the underlying discrete events associated with plastic deformation/in loaded flexures.

PACS numbers: 07.10.Pz 73.40.-c 05.40.-a 04.80.Nn 07.30.-t 95.55.Ym 07.60.Ly

Submitted to: Class. Quantum Grav.

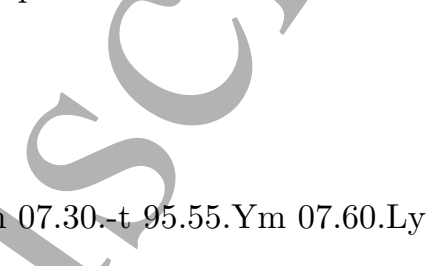




\section{Introduction}

Intermediate upgrades to advanced interferometric gravitational wave detectors such as Advanced LIGO (aLIGO) [1] are envisaged before the realisation of thirdgeneration detectors such as the Einstein Telescope (ET) [2]. Given the first detections of gravitational waves being associated with the merger of binary black hole systems [3], there is continued interest in enhancements at the low frequency band of the current detectors $(10 \mathrm{~s}$ of $\mathrm{Hz}$ ), to allow searches for coalescing intermediate black hole binary systems. Gravitational wave detectors operate as modified Michelson interferometers, with kg-scale test masses suspended at the end of kmscale arms. Improvements at low frequency will require a reduction in radiation pressure noise (achievable by increasing the mass of the test masses), reduction in the final-stage suspension thermal noise and the vertical component of thermal noise, and improvements in seismic isolation. This paper investigates technology that could significantly reduce the vertical thermal noise component within an enhanced Advanced LIGO configuration operating at room temperature, through use of silicon blade springs with protective diamond-like carbon (DLC) coatings.

Another relevant noise is associated with how elastic materials respond to changing external forces through small (discrete) changes in stress, rather than a continuously smooth deformation [4], often referred to as crackling noise. Models for describing the current maraging steel cantilevers within aLIGO are under development [5, 6]. However, replacing the maraging steel with silicon blade springs is expected to significantly reduce the total number of crackling events due to the reduction of atomic creep (e.g. dislocation slip and climb, grain boundary sliding, and diffusional flow).

It is well-established that DLC, a wide class of amorphous carbons containing a proportion of $20-90 \% \mathrm{sp}^{3}$ carbon to carbon (C-C) bonding, as well as, in some cases, incorporating some quantity of hydrogen [7, 8, 9, 10, 11], is highly compatible with silicon as a coating material [12, 13, 14]. The adhesion of DLC coatings to silicon substrates is generally excellent, with silicon often being used as an adhesion layer and/or dopant to improve DLC bonding to substrates on which DLC would not otherwise adhere [13, 14, 15, 16]. Such coatings continue to find a very wide range of applications, including for protective (e.g. tribological) [9, 16, 17, 18] and optical (particularly mid-IR) purposes [9].

While the exact properties of any DLC coating depend on the film deposition 
method used and on the composition and structure of the coating, in general it can be said that that DLCs are characterised by high hardness [9, 16, 15], low coefficient of friction [9, 19], excellent abrasion resistance [19], and transparency in the mid-infrared [8]. In future detector designs utilising silicon as a construction material, DLC may therefore be an interesting coating material for improvement of the robustness of suspension components, e.g. the cantilever blade springs [20].

A thermally-grown oxide coating, prepared by oxidation of silicon in wet $\mathrm{N}_{2}$ at $1000^{\circ} \mathrm{C}$ has previously been reported to significantly improve the tensile strength of silicon flexures [3]. For comparison with these findings, we have performed oxide growth under the same conditions. Since it is unclear whether the oxide coating is responsible for the improvement in strength of silicon, or whether this is effectively due to heat treatment, for further comparison we have also deposited silica coatings by microwave-assisted magnetron sputtering at room temperature. This may allow further elucidation of the mechanism of the improvement in strength by thermal oxidation.

\section{Experimental methods}

\subsection{Substrate preparation}

Silicon flexures were prepared by mechanically dicing $0.5 \mathrm{~mm}$ thick $<100>$ P-type (boron-doped) DSP (double-side polished) silicon wafers, with resistivity $1-10 \Omega \mathrm{m}$ (University Wafer, USA), into strips of dimensions $30 \times 3.4 \mathrm{~mm}$, using a Disco DAD3230 dicing saw system. Half of the flexures were mechanically polished $(3 \mu \mathrm{m}$ aluminium oxide compound) on the long edges to assess the effect of edge quality on tensile strength; this resulted in average strip width of $3.2 \mathrm{~mm}$.

\subsection{Deposition of DLC coatings}

Thick single-layer $(1.00 \pm 0.05 \mu \mathrm{m})$ and multilayer $(3.13 \pm 0.02 \mu \mathrm{m})$ DLC films were deposited on the silicon flexures using an IC 10000 pulsed-DC hollow cathode PECVD (plasma-enhanced chemical vapour deposition) system (Sub-One Systems, Tucson, AZ, USA), as shown in Figure 1. This process and system are described at length elsewhere [15, 16]. Briefly, the process allows deposition of increased thickness (up to tens of microns in total) modified (graded multilayer) DLC films as compared to traditional PECVD or PVD (physical vapour deposition) methods 


\section{Coatings and surface treatments for enhanced performance suspensions}

(typically limited to around 1-2 $\mathrm{m}$ due to high intrinsic compressive stress of the order of -0.5 to $-2.0 \mathrm{GPa}$ ) [9, 21], at high film growth rates of the order of $300 \mathrm{~nm} / \mathrm{min}$. This is achieved by using a graded multilayer structure, typically using siliconcontaining precursors to firstly deposit a silicon-rich amorphous hydrogenated (a$\mathrm{Si}: \mathrm{C}: \mathrm{H})$ adhesion layer, and by doping the subsequent layers with progressively decreasing quantity of silicon, finally depositing a cap layer of pure amorphous hydrogenated carbon (a-C:H). As this is a non-line-of-sight PECVD process, a wide range of substrate geometries may be accommodated, including interior surfaces.
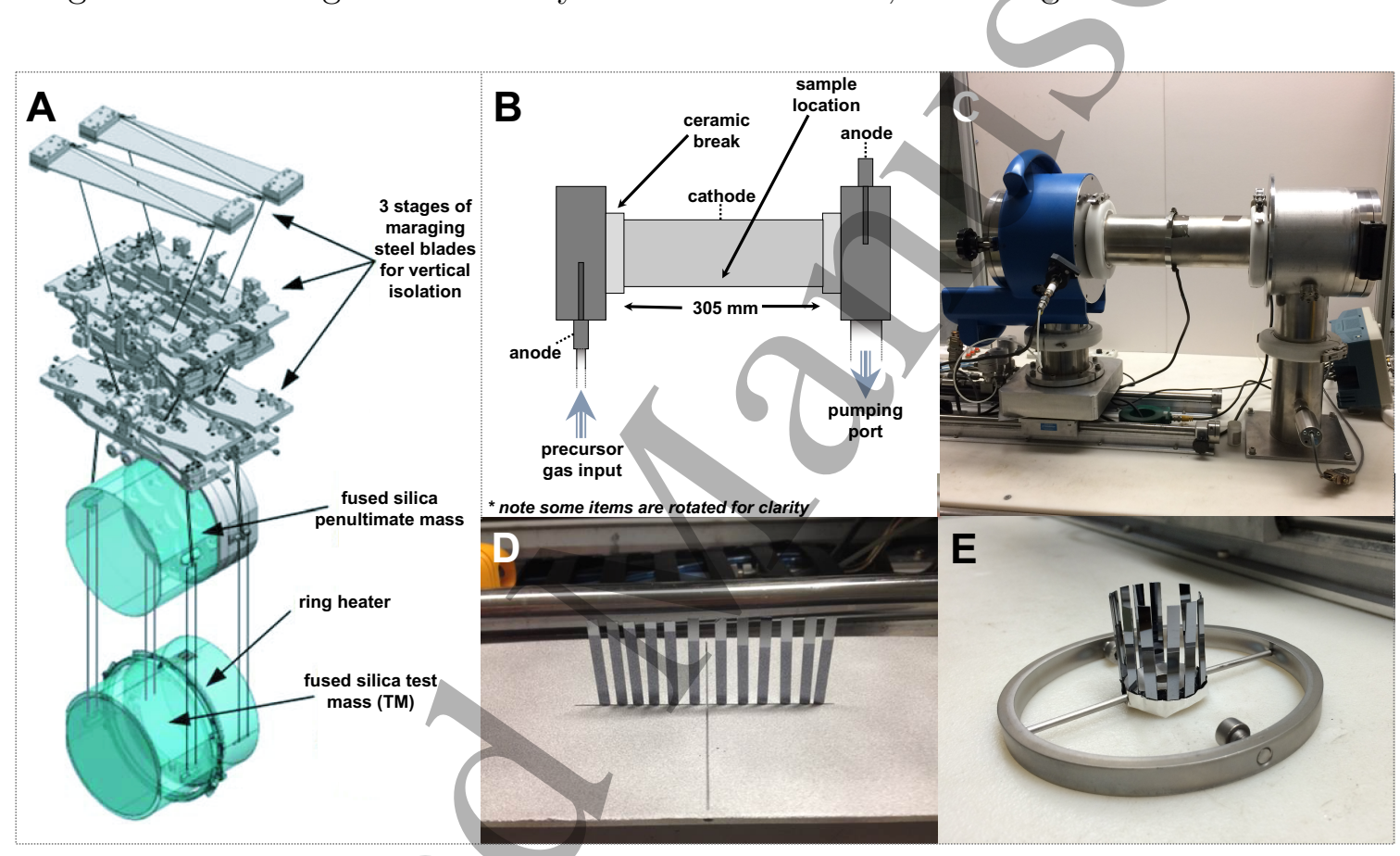

Figure 1: (A) Schematic of the current Advanced LIGO suspension system [22]. Schematic (B) and picture (C) of the pulsed-DC hollow cathode PECVD system used to deposit DLC coatings and perform plasma etch treatment on silicon flexures. Samples were mounted in a vertical position (D) and horizontal position (E) in the centre of the cathode volume.

All DLC coatings, except sample set 19, were deposited in vertical parallel orientation using an aluminium stage within a $100 \mathrm{~mm}$ diameter pipe chamber, as shown in Figure $1 \mathrm{C}$. Sample set 19 was coated with the strips mounted in a horizontal 
Coatings and surface treatments for enhanced performance suspensions

Table 1: Details of deposition parameters for DLC coatings and argon plasma treatment. Single-layer DLC coatings follow steps 1-3 and 9-10. Multilayer DLC coatings follow all steps. Extended argon plasma treatment samples follow steps 1-3.

\begin{tabular}{llllllll}
\hline $\begin{array}{l}\text { Step } \\
\text { Ar } \\
(\mathrm{sccm})\end{array}$ & $\begin{array}{l}\mathrm{H}_{2} \\
(\mathrm{sccm})\end{array}$ & $\begin{array}{l}\mathrm{C}_{2} \mathrm{H}_{2} \\
(\mathrm{sccm})\end{array}$ & $\begin{array}{l}\text { TMS } \\
(\mathrm{sccm})\end{array}$ & $\begin{array}{l}\text { Pressure } \\
(\mathrm{mTorr})\end{array}$ & $\begin{array}{l}\text { Time } \\
(\mathrm{min})\end{array}$ & $\begin{array}{l}\text { Power } \\
(\mathrm{DC} 1 / \mathrm{DC} 2, \mathrm{~kW})\end{array}$ \\
\hline 1 & 50 & 100 & - & - & 70 & $0: 30$ & $0.035 / 0.180$ \\
2 & 50 & 100 & - & - & 70 & $10: 00$ & $0.040 / 0.200$ \\
3 & 100 & - & - & - & 70 & $30: 00 / 60: 00$ & $0.040 / 0.200$ \\
& & & & & & $($ extended etch) & \\
4 & 100 & - & - & - & 70 & $0: 20$ & $0.040 / 0.200$ \\
5 & 90 & - & - & 70 & 120 & $3: 30$ & $0.040 / 0.144$ \\
6 & 60 & - & 20 & 60 & 70 & $3: 30$ & $0.040 / 0.216$ \\
7 & 50 & - & 40 & 40 & 70 & $3: 30$ & $0.052 / 0.136$ \\
8 & 50 & - & 80 & 20 & 70 & $3: 30$ & $0.052 / 0.136$ \\
9 & 50 & - & 120 & - & 90 & $5: 00$ & $0.052 / 0.136$ \\
10 & $85\left(+200 \mathrm{~N}_{2}\right)$ & - & - & - & - & $2: 00$ & $0.000 / 0.000$ \\
\hline
\end{tabular}

orientation on a ring fitting as shown in Figure 1D, in order to assess the effect of substrate orientation within the plasma chamber.

Deposition parameters were as shown in Table 1, with one group of samples being treated with a standard argon plasma etch prior to coating deposition, and a second set being subjected to an extended argon plasma etch. Additionally, sample set 18 was coated with multilayer DLC, following thermal oxidation of the silicon substrate, as described later in the paper.

\subsection{Deposition of magnetron sputtered silica}

Sample sets 7,8, 14 and 15 were coated with silica in two separate deposition runs (one for each side of the silicon strips), and mounted at a $45^{\circ}$ angle with respect to the silicon target to enable coating across all surfaces. The system used was a DSI MicroDyn microwave-assisted sputtering unit. Coatings were deposited by sputtering from a silicon target in oxygen atmosphere, resulting in coating thickness of $542 \pm 7 \mathrm{~nm}$. 


\subsection{Thermal oxidation of silicon substrates}

Sample sets 17 and 18 were wet oxidised in saturated $\mathrm{N}_{2}$ atmosphere for 1 hour at $1000^{\circ} \mathrm{C}$, resulting in an oxide layer being grown on all silicon surfaces, of layer thickness $275 \pm 1 \mathrm{~nm}$. Sample set 18 was then overcoated with single-layer DLC of thickness $1 \mu \mathrm{m}$ on all surfaces, by the same method as used for sample set 3 .

\subsection{Tensile strength testing}

Silicon is a brittle material, and does not withstand significant plastic deformation under stress, thus making it prone to shatter [23, 24]. Previous tensile strength studies on silicon samples have shown that silicon will fail/at tensile stress of approximately $200 \mathrm{MPa}$ at room temperature [25]. In [23] it was also shown that fracture or failure stress decreases with increasing silicon sample cross-sectional area under three-point flexural testing. More recently, Cumming et al. [26] reported tensile strengths of the order of $140 \mathrm{MPa}$ for untreated silicon, and demonstrated significant improvements in strength via various surface treatments. The highest tensile strength was achieved by wet oxidising $<100>$ silicon, showing a maximum average figure of around $300 \mathrm{MPa}$, with some individual strengths up to $450 \mathrm{MPa}$.

It is believed that the decreasing strength with increasing cross-sectional area is explained by localised regions of high intrinsic stress [25] e.g. Griffith cracks [27] on the surface and edges of the sample, and bulk defects such as dislocations and impurities such as oxygen [23, 28]. Table 2 summarises the relevant properties of the silicon samples used in this study. A total of 182 silicon samples were tested for tensile strength, including "base" or untreated, and silicon with 9 different variations of surface treatments, both with and without mechanical polishing of the ribbon edges. Additionally, the effect of orientation of silicon flexures within the vacuum chamber was investigated as discussed earlier and shown in Figure 1C and Figure 1D.

In order to avoid damaging the surface of the samples and prevent slippage during testing, all samples were bonded between two aluminium "fuse end" attachments, using Araldite 2012 2-part epoxy adhesive, as detailed in [26]. Sample assemblies were then bolted via a drilled hole in each fuse end to an alignment jig to ensure correct alignment of the assemblies before curing; the adhesive was cured by placing the sample jigs on a hotplate at $80^{\circ} \mathrm{C}$ for 1 hour, followed by a 24 hour room temperature curing. After curing, samples were mounted on an adaptable tensile strength testing system [26, 29] as shown in Figure 2A, with universal joints installed 
Table 2: Details of silicon samples used in tensile and flexural strength tests.

\begin{tabular}{|c|c|c|c|}
\hline Sample set & $\begin{array}{l}\text { Dimensions* } \\
(\mathrm{mm})\end{array}$ & Edge quality & Surface treatment \\
\hline 1 & $30.0 \times 3.4 \times 0.5$ & Unpolished & None \\
\hline 2 & $30.0 \times 3.4 \times 0.5$ & Unpolished & Extended argon etch \\
\hline 3 & $30.0 \times 3.4 \times 0.5$ & Unpolished & $1 \mu \mathrm{m}$ single-layer DLC \\
\hline 4 & $30.0 \times 3.4 \times 0.5$ & Unpolished & $\begin{array}{l}\text { Extended argon etch and } \\
1 \mu \mathrm{m} \text { single-layer DLC }\end{array}$ \\
\hline 5 & $30.0 \times 3.4 \times 0.5$ & Unpolished & 3.1 $\mathrm{m}$ multilayer DLC \\
\hline 6 & $30.0 \times 3.4 \times 0.5$ & Unpolished & $\begin{array}{l}\text { Extended argon etch and } \\
3.1 \mu \mathrm{m} \text { multilayer DLC }\end{array}$ \\
\hline 7 & $30.0 \times 3.4 \times 0.5$ & Unpolished & $542 \mathrm{~nm}$ sputtered silica \\
\hline 8 & $30.0 \times 3.4 \times 0.5$ & Unpolished & Extended argon etch and \\
\hline 9 & $30.0 \times 3.2 \times 0.5$ & Mechanically polished & None \\
\hline 10 & $30.0 \times 3.2 \times 0.5$ & Mechanically polished & Extended argon etch \\
\hline 11 & $30.0 \times 3.2 \times 0.5$ & Mechanically polished & $1 \mu \mathrm{m}$ single-layer DLC \\
\hline 12 & $30.0 \times 3.2 \times 0.5$ & Mechanically polished & $\begin{array}{l}\text { Extended argon etch and } \\
1 \mu \mathrm{m} \text { single-layer DLC }\end{array}$ \\
\hline 13 & $30.0 \times 3.2 \times 0.5$ & Mechanically polished & $3.1 \mu \mathrm{m}$ multilayer DLC \\
\hline 14 & $30.0 \times 3.2 \times 0.5$ & Mechanically polished & $\begin{array}{l}\text { Extended argon etch and } \\
3.1 \mu \mathrm{m} \text { multilayer DLC }\end{array}$ \\
\hline 15 & $30.0 \times 3$. & Mechanically polished & $542 \mathrm{~nm}$ sputtered silica \\
\hline 16 & $30.0 \times 3$ & Mechanically polished & $\begin{array}{l}\text { Extended argon etch and } \\
542 \mathrm{~nm} \text { sputtered silica }\end{array}$ \\
\hline 17 & & Unpolished & $275 \mathrm{~nm}$ wet oxidised silica \\
\hline 18 & $30.0 \times$ & Unpolished & $\begin{array}{l}275 \mathrm{~nm} \text { wet oxidised silica } \\
\text { plus } 1 \mu \mathrm{m} \text { single-layer DLC }\end{array}$ \\
\hline 19 & $30.0 \times 3.4 \times 0.5$ & Unpolished & $\begin{array}{l}3.1 \mu \mathrm{m} \text { multilayer DLC, } \\
\text { horizontally mounted }\end{array}$ \\
\hline
\end{tabular}

* Approximate dimensions: Individual samples showed slight variation in width, owing to the dicing and/or polishing process; this is accounted for in the failure stress calculations. 
above and below the sample assembly to minimise bending/tearing. Furthermore, the lower fuse end was free to rotate, to reduce torsional forces on the sample. Samples were tested to destruction, with breaking stress calculated by $\sigma=F / A$, where $A$ is cross-sectional area of the sample, and $F$ is the maximum force exerted on the sample, i.e. the force at which failure occurs.
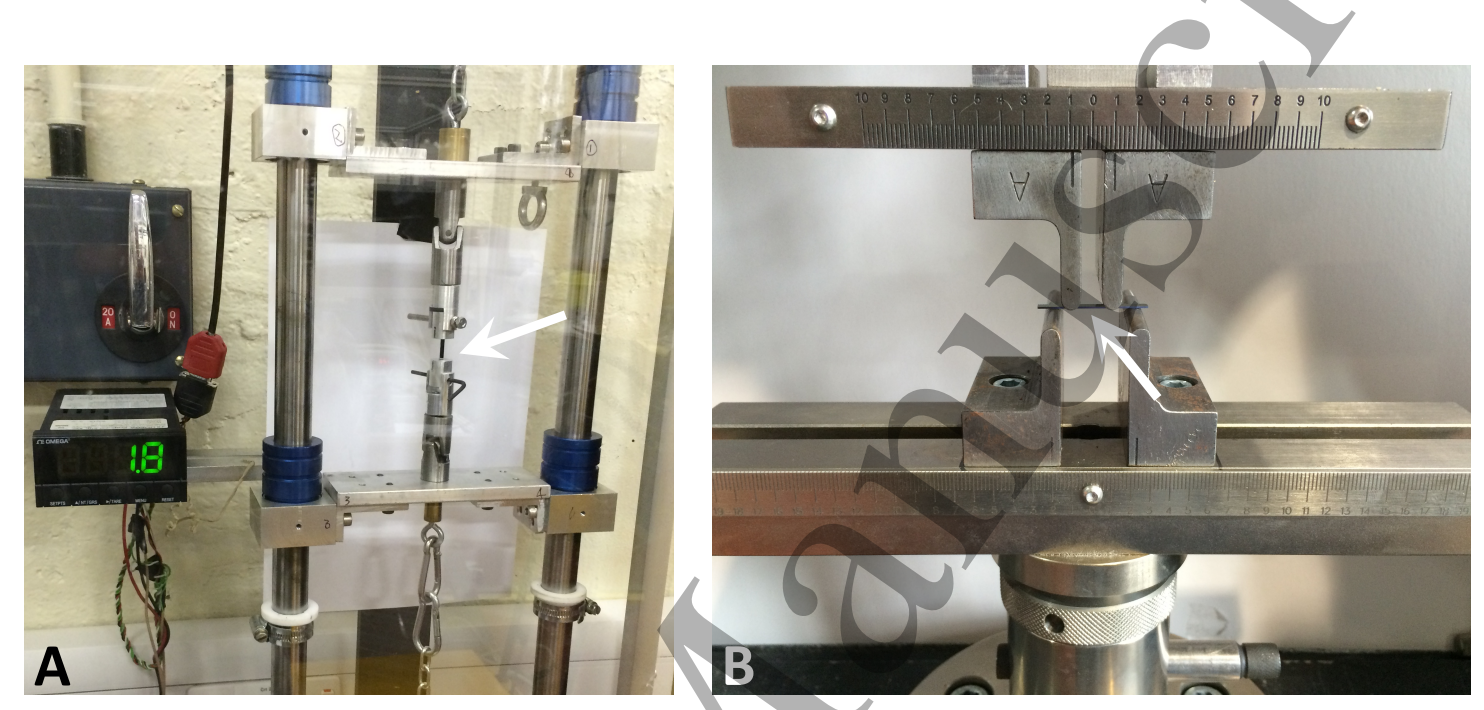

Figure 2: Image of (A) tensile and (B) 4-pointflexural strength testing set ups, with arrows indicating sample location.

\subsection{Four-point flexural strength testing}

3-point bending strength test measurements were previously made on fine silicon whisker samples by Pearson [23]; fracture stresses of approximately 1-2 GPa, with a maximum fracture stress of $5 \mathrm{GPa}$ were reported for samples of 20-100 $\mu \mathrm{m}$ diameter. As already discussed, evidence was presented that fracture stress decreases significantly with increasing cross-sectional area of the silicon sample. These samples were smaller in cross-sectional area than would be required for silicon ribbons/fibres or blade springs for future gravitational wave detectors (which are likely to be of the order $0.25-10 \mathrm{~mm}^{2}$ ) [26].

4-point bending tests are similar to 3-point bending tests, with the major difference between the methods being the location of the bending moment. The 
four point bending method allows for a more uniform distribution between the two loading noses, whereas the three point bending method's stress is located under the single loading nose. In the case of brittle materials such as silicon, four point bend testing is generally preferred when determination of strength for design purposes is required [30, 31, 32]. This is because the centre span is uniaxially stressed, and so no shear stresses exist, therefore the sample is subjected to both compressive and tensile stresses, and it is typical for measured flexural strength to be greater than tensile strength [33]. However, 4-point flexural strengths are likely to be smaller than those measured by 3-point flexural testing, since a much larger portion of the sample is exposed to the maximum applied stress [30, 32].

This method may be more appropriate for examining the effects of surface treatments on silicon components for gravitational wave detectors than tensile testing, particularly in the case of silicon blade spring cantilevers [20].

Flexural strength testing was carried out on sample sets 1-8, 17 and 18, i.e. only samples with unpolished edges, with a total of 96 samples tested. Flexural tests were conducted using a universal testing machine (model Instron $596650 \mathrm{kN}$ ) with crosshead speed of $0.25 \mathrm{~mm} / \mathrm{min}$. Cylindrical profile loading noses were set up with a support span of $21 \mathrm{~mm}$ and loading span of $7 \mathrm{~mm}$, as shown in Figure $2 \mathrm{~B}$.

Calculation of failure stress was carried out according to the equation for 4-point flexural testing on rectangular cross-sections [30, 31, 32] with loading span one third the size of the support span, such that,

$$
\sigma=\frac{F L}{b d^{2}}
$$

where $\sigma$ is stress at the point of rupture/failure, $F$ is applied force at point of rupture, $L$ is the width of support span, $b$ is width of test sample, and $d$ is thickness of sample.

\subsection{Abrasion testing}

Single- and multilayer DLC coatings on $2 \times 2 \mathrm{~cm}$ silicon $<100>$ substrates, both with and without extended Ar pre-etch, together with untreated base silicon (corresponding to sample sets 3, 4, 5, 6 and 1 respectively), were subjected to the TS1888 [34] test using a Grittington abrasion test rig. Although a military standard test method, TS1888 is widely used in the optical coating industry for qualitative assessment of abrasion resistance of optical coatings. Samples were subjected to 
5 minutes of wiping at a setting of $1000 \mathrm{rpm}$, and a wiper load of $20 \mathrm{~g}$, using a mixture of $1 \mathrm{~cm}^{3}$ of sand to DEFSTAN 07-55 Type C (military standard) in $10 \mathrm{ml}$ of water. The sample is considered to have acceptable abrasion resistance and to have passed the test if there are no significant signs of scratching or other surface imperfections after the test. Samples were tested as per TS1888 5.4.3 and visually inspected. Scanning electron microscope images were also recorded of the/abraded samples, using a Hitachi S-4100 FE SEM.

\section{Results and analysis}

\subsection{Tensile strength testing}

The results for tensile strength testing of all 19 sample sets are shown in Figure 3 .

3.1.1. Unpolished-edge silicon flexures Base (i.e. untreated) silicon (set 1) shows an average failure stress of $225 \pm 16 \mathrm{MPa}$, with the argon plasma-treated set 2 showing similar strength of $221 \pm 31 \mathrm{MPa}$ (all errors quoted as standard error of the mean). Coating with $1 \mu \mathrm{m}$ of single-layer DLC (set 3) improves the average strength by around $65 \%$ to $362 \pm 53 \mathrm{MPa}$; adding an extended Ar pre-etch before deposition (set 4) improves the average strength further still, to $405 \pm 33 \mathrm{MPa}$, which is the highest average tensile strength measured in this study. However, these latter two results are within error of one another, so it is unclear as to how significant an effect the extended etch has.

Multilayer DLC (set 5) improves average failure stress of base silicon by $35 \%$ to $304 \pm 32 \mathrm{MPa}$; and, again, combined with an extended duration argon plasma etch (set 6) increases the average strength to $353 \pm 45 \mathrm{MPa}$. Again, these two results are within error of one another.

All of the magnetron silica-coated samples' strengths were confined to a fairly low and narrow range, with broadly similar values to the low outlying data points for other sample types, but without the high outliers seen with DLC coating. There is no evidence in this study that magnetron-sputtered silica coating (set 7) can enhance the strength of silicon ribbon; rather, the average strength is reduced to $87 \pm 16 \mathrm{MPa}$, with the argon-etched counterpart (set 8) having similar strength of $93 \pm 11 \mathrm{MPa}$. Again, the influence of extended argon plasma pre-etch is not clear. The lower than expected strength of these samples may have been caused by the mounting 


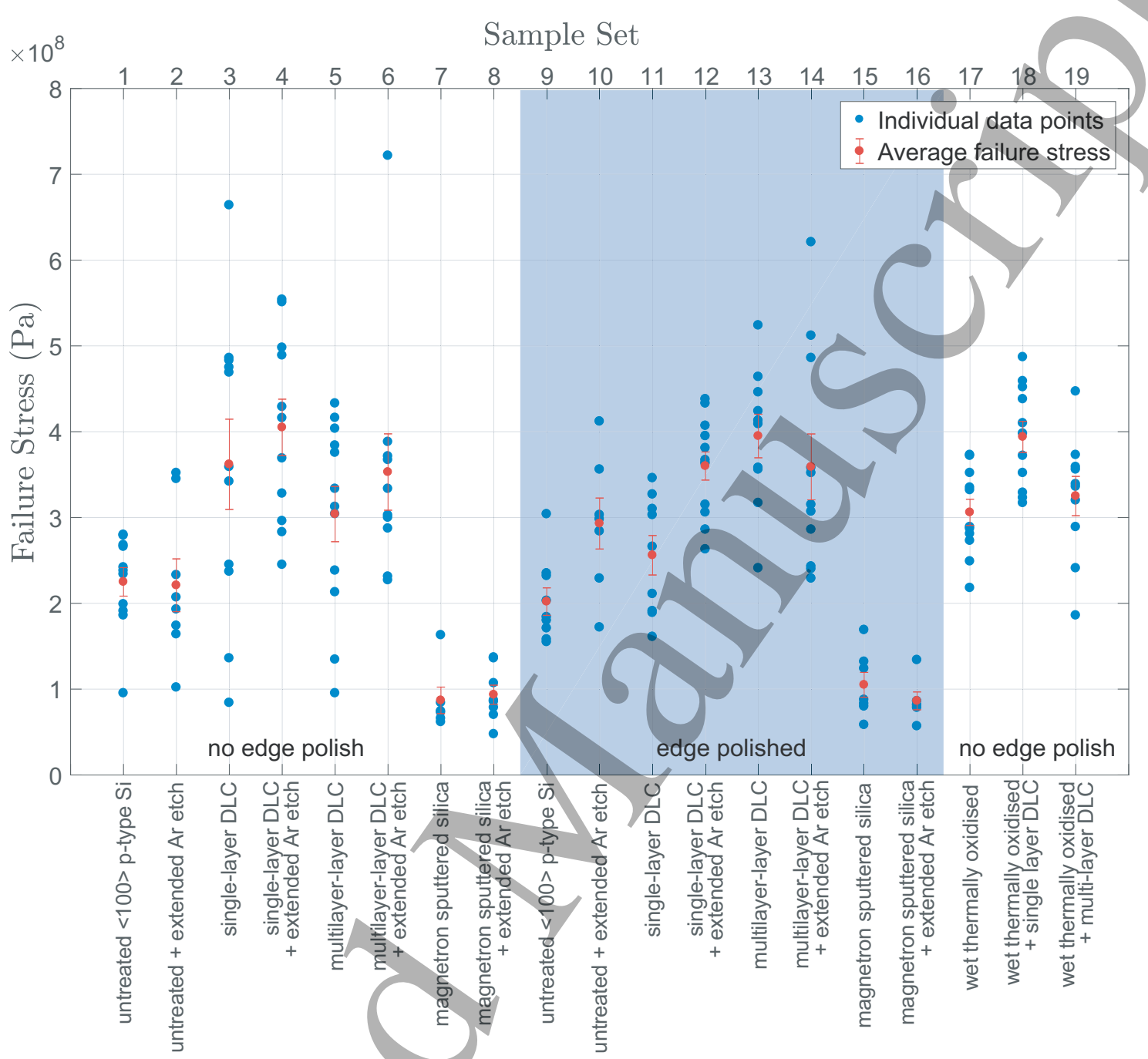

Figure 3: Tensile strength test results for sample sets 1-19

and dismounting procedure. Samples were held in place for deposition using doublesided conductive carbon tape; these proved to be rather difficult to remove from the mounting plates after deposition, and it seems likely that the physical removal process created additional surface defects, which may have resulted in lower failure strengths. It is, however, also worthy of note that the range of strengths measured for these samples is rather narrower than for other sample types, which would not necessarily be expected were the strengths only being limited by random physical 


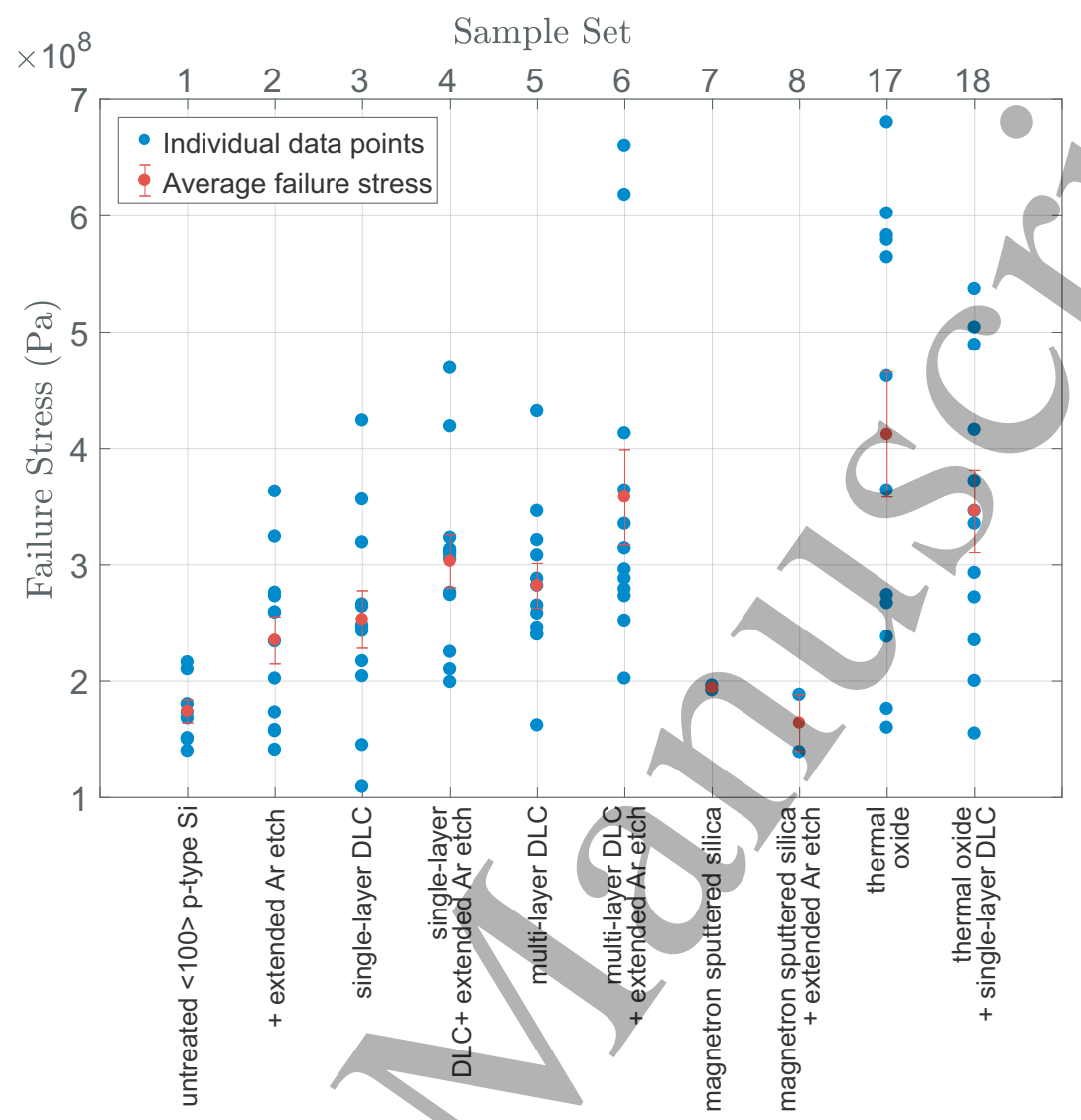

Figure 4: Flexural strength test results for sample sets 1-8, 17 and 18

defects caused by dismounting.

By contrast, thermally-grown silica coating (set 17) was shown to increase the average breaking strength of base silicon by around $35 \%$ to $306 \pm 15 \mathrm{MPa}$; addition of a $1 \mu \mathrm{m}$ single-layer DLC coating on top of thermal oxide (set 18) improves the average strength further to $394 \pm 18 \mathrm{MPa}$.

It is therefore quite difficult to draw a firm conclusion explaining the strengthening effect of the thermal oxidation of silicon; this process certainly improves the strength of base silicon, but whether this is due to the material being heated to $1000^{\circ} \mathrm{C}$ or to the oxide coating itself is unclear at this point. It was hoped that magnetron silica coatings would have elucidated the point; however, the unexpectedly low strengths of these samples, as discussed previously, prevented this. 
With regard to the orientation of silicon ribbon samples within the vacuum chamber, we can compare sample sets 5 and 19. Both sets were coated with $3.1 \mu \mathrm{m}$ thick multilayer DLC on unpolished edge silicon, with standard argon plasma preetch. Sample set 5 was mounted in the standard way; vertically, seated in a slot on the sample stage, and oriented parallel to the pipe length, as shown in Figure $1 \mathrm{C}$. Set 19 was mounted horizontally and radially on a ring fitting within the pipe, parallel to the direction of precursor gas flow, as shown in Figure 1D.

Sample set 19 showed a very slight increase in average tensile failure stress, at $325 \pm 23 \mathrm{MPa}$, compared to $304 \pm 32 \mathrm{MPa}$ for set 5; as these results are within standard error of one another, it can be concluded that tensile strength/shows no strong dependence here on orientation within the vacuum chamber. It is also interesting to note that film thicknesses were 3.10 and $3.13 \mu \mathrm{m}$ for the vertical and horizontal mounts respectively; again, within experimental error.

3.1.2. Polished-edge silicon flexures Base silicon with polished edges (set 9) exhibited an average failure stress of $202 \pm 16$ MPa, lower than that of unpolished silicon; however, combined with an extended argon plasma pre-etch (set 10) the strength is somewhat improved to $293 \pm 29 \mathrm{MPa}$. The standard etch (30 mins) corresponds to $261 \mathrm{~nm}$ surface removal, and the extended etch (60 mins) corresponds to $522 \mathrm{~nm}$ surface removal.

$1 \mathrm{\mu m}$ thick single-layer DLC improved the strength of polished edge silicon flexures (set 11) to $256 \neq 23 \mathrm{MPa}$; combined with an extended pre-etch (set 12), this is increased to $360 \pm 16 \mathrm{MPa}$.

$3.1 \mu \mathrm{m}$ thick multilayer DLC coating (set 13) resulted in an average tensile strength of $395 \pm 25 \mathrm{MPa}$, which was the highest average figure measured for coated polished-edge silicon, an increase of around $95 \%$ as compared to base silicon with mechanically polished edges. This was also higher than that of the corresponding unpolished sample set. In contrast to the unpolished analogues, adding an extended argon pre-etch step (set 14) resulted in an average decrease in strength compared to standard etch to $359=38 \mathrm{MPa}$, albeit within experimental error of the result for set 13.

As with sample sets 7 and 8, $542 \mathrm{~nm}$ thick magnetron sputtered silica coatings on polished edge silicon showed a large decrease in average tensile strength compared to untreated silicon, to $105 \pm 14 \mathrm{MPa}$ and $86 \pm 10 \mathrm{MPa}$ for standard (set 15) and extended argon etch (set 16) respectively. Again, it seems likely that the mounting and 
dismounting process for the magnetron silica coatings, which necessitated mechanical removal of the samples from the mounting tape, may have been responsible for the lower than expected tensile strengths measured.

\subsection{Four-point flexural strength testing}

All samples tested for flexural strength were silicon flexures with unpolished edges; results for all samples are shown in Figure 4. In most cases, measured flexural strengths were similar to, or slightly lower than, tensile strengths.

Base silicon (set 1) showed flexural strength of $174 \pm 10 \mathrm{MPa}$, while applying an argon plasma etch increased the figure to $235 \pm 20 \mathrm{MPa}$.

As with tensile strength, single-layer DLC (set 3) appears to improve the flexural strength of silicon ribbon to $253 \pm 25 \mathrm{MPa}$; and, combined with argon pre-etch (set 4 ), the average figure increased to $303 \pm 23 \mathrm{MPa}$.

Multilayer DLC (set 5) exhibited average flexural strength of $282 \pm 19 \mathrm{MPa}$ and, again, argon pre-etch plus multilayer DLC (set 6) increased the strength further to $358 \pm 41 \mathrm{MPa}$.

Interestingly, in contrast to the case of tensile strength measurements, magnetron sputtered silica-coated silicon was found to have a similar flexural strength to that of base silicon. However, in the case of both sets 7 and 8, this finding was based on two data points owing to limited numbers of samples, and so the data must be treated with caution here.

Thermally-grown silica coating (set 17) was shown to significantly increase the average flexural strength of base silicon, to $412 \pm 54 \mathrm{MPa}$, while addition of a $1 \mathrm{\mu m}$ single-layer DLC coating on top of the thermal oxide (set 18) showed a lower average figure of $346 \pm 36 \mathrm{MPa}$, so within experimental error of thermal oxide alone.

\subsection{Abrasion testing}

It is noteworthy that the uncoated silicon sample showed visible damage to the surface after 5000 wiper revolutions; scratches were clearly visible to the naked eye. All of the coated samples passed the TS1888 test protocol, meaning that they showed no visible damage after 5000 revolutions.

While by its nature a qualitative pass/fail test, the effectiveness of both singleand multilayer DLC coatings as protective layers for silicon components was clearly demonstrated. Figure 5 shows SEM images of each of the 5 samples tested. 
Coatings and surface treatments for enhanced performance suspensions

For untreated silicon, the surface is noticeably degraded by the wiper test; deep scratches are apparent. The single-layer DLC coated sample shows no surface degradation, although a pinhole defect (caused by a local electrical breakdown during the deposition process) is apparent. There are occasional visible nodular asperities in the SEM images of both single-layer DLC coated silicon with extended plasma etch; such features are typical of PECVD-deposited amorphous carbons [15, 35, 36.
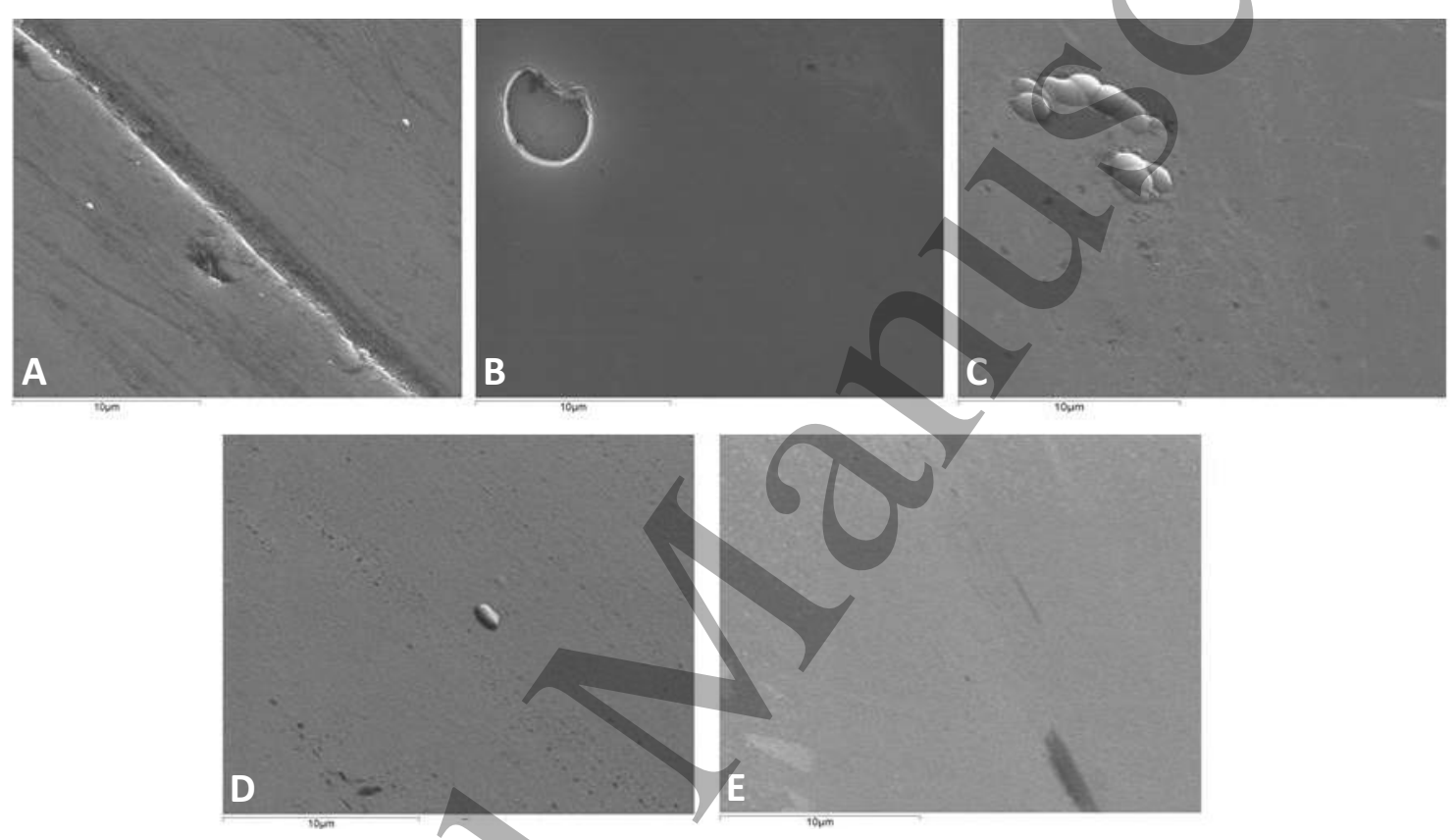

Figure 5: SEM images of samples abraded per TS1888 method for (A) uncoated silicon, (B) single-layer DLC (set 3), (C) single-layer DLC with extended pre-etch (set 4), (D) multilayer DLC (set 5), (E) multilayer DLC with extended pre-etch (set 6 ). Note the damage to the uncoated silicon. The other surface features are typical of amorphous carbon coatings.

\subsection{Thermal noise}

As discussed, further reductions in the longitudinal thermal noise associated with the fused silica monolithic suspensions in Advanced LIGO will likely require improvements in the thermal noise performance of the lower cantilever blade springs. 
These are currently fabricated from maraging steel, of $4.2 \mathrm{~mm}$ thickness and loss factor of $1 \times 10^{-4}$ in addition to relevant thermoelastic contribution [37. The geometry for enhanced silicon blade springs will be determined from the thermal noise performance and the ultimate breaking strength. For simplicity, scaling the thickness and keeping the remaining blade geometries, and providing a factor of 50 safety margin in breaking strength (where $\sigma_{\text {failure }}=350 \mathrm{MPa}$ ), would allow/a silicon blade thickness of $1.4 \mathrm{~mm}$ to be utilised. The vertical thermal noise can be shown to be related to the harmonic motion such that,

$$
x_{\text {vertical }}=\sqrt{\frac{4 k_{\mathrm{B}} T \phi_{\mathrm{tot}} \omega_{\mathrm{v}}^{2}}{m \omega\left(\omega_{\mathrm{v}}^{2}+\omega^{2}\right)^{2}+\left(\phi_{\mathrm{tot}} \omega_{\mathrm{v}}^{2}\right)^{2}}},
$$

where $k_{\mathrm{B}}$ is Boltzmanns constant, $T$ is the temperature, $\phi_{\text {tot }}$ is the total mechanical dissipation, $m$ is the pendulum mass, $\omega_{\mathrm{v}}$ is the vertical bounce mode angular frequency, and $\omega$ is the angular frequency 38. The vertical motion of the suspension stack will couple to the horizontally sensed displacements of the interferometer by a coupling factor of 1/1000 (due to the curvature of the Earth [39]). The mechanical dissipation of the DLC coatings was investigated by measuring silicon cantilever resonators with applied DLC coatings [40, 41]. The mechanical dissipation of the uncoated cantilevers was observed to follow the expected thermoelastic dissipation, as previously reported for similar geometry samples [40] as shown in Figure 6.

The mechanical dissipation of cantilevers was measured before and after coating, and the mechanical dissipation of the DLC coating, $\phi_{\text {DLC }}$, calculated by scaling the difference with the ratio of strain energies in the coating and substrate, $E_{\text {substrate }} / E_{\text {coating }}$ such that,

$$
\phi_{\text {DLC }}=\frac{E_{\text {substrate }}}{E_{\text {coating }}}\left(\phi_{\text {coated }}-\phi_{\text {uncoated }}\right) .
$$

The Youngs moduli are taken to be $E_{\text {coating }}=110 \mathrm{GPa}$ [15] and $E_{\text {substrate }}=$ $161 \mathrm{GPa}$ [40]. Coatings were deposited at different thicknesses, to evaluate any significant thickness dependence in the loss. Two cantilevers were coated in the same coating run but with one cantilever fully exposed, to coat on all sides, and the other placed on an aluminium platform, to coat on the upper surfaces only. Due to the large compressive stresses, typically of the order of $-0.8 \mathrm{GPa}$ for singlelayer a-C:H deposited by hollow cathode PECVD [15], the single- and double-side 

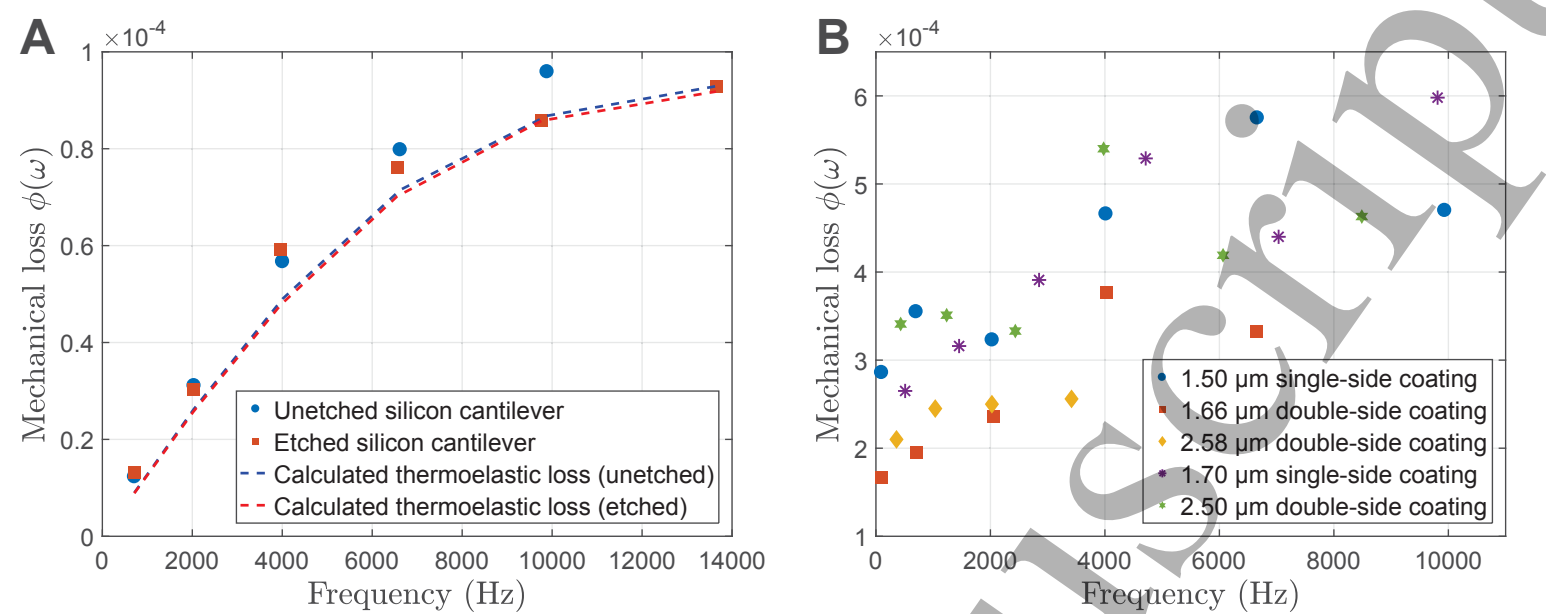

Figure 6: (A) Measured mechanical loss for an uncoated silicon cantilever, with and without Ar etch, compared to the calculated thermoelastic dissipation. The thickness of the cantilever was calculated from the resonant frequencies to be $97.8 \pm 0.4 \mu \mathrm{m}$ and $96.7 \pm 0.3 \mu \mathrm{m}$ before and after etching respectively. (B) Calculated mechanical loss values for DLC coatings on silicon cantilevers. The standard errors are too small to show on the plots, typically being approximately $1 \%$ from a series of 3 to 4 subsequent ringdowns.

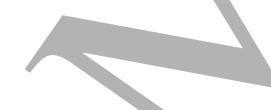

coated cantilevers will provide evidence of whether there is a stress dependence in the mechanical dissipation. Figure 6 shows the compiled losses for these three cantilevers, alongside SEM cross-sectional images of the deposited coatings. An upper limit of $\phi_{\mathrm{DLC}}=5 \times 10^{-4}$ is taken for the subsequent thermal noise calculations.

The mechanical dissipation of the modelled silicon blade springs was calculated using the upper limit value of $\phi_{\text {DLC }}$ (assuming $1 \mu \mathrm{m}$ thick DLC coating and scaled again by the energy ratio for a $1.4 \mathrm{~mm}$ thick blade spring). Figure 7 shows the final calculated vertical thermal noise, compared to the current Advanced LIGO design, calculated using the GWINC (Gravitational Wave Interferometer Noise Calculator) 42. In this case, the contribution from the final monolithic stage is removed, since this requires improvements. Proposed room temperature upgrades for Advanced LIGO are discussed in detail by Hammond et al. [43], which could include heavier test masses $(40 \rightarrow 160 \mathrm{~kg})$ in addition to longer $(0.6 \rightarrow 1.1 \mathrm{~m})$ and thinner silica suspensions with improved mechanical loss and dilution factors [44]. 
A 6.4-times reduction in the vertical thermal noise is observed at $10 \mathrm{~Hz}$, with improvement across all frequencies above $3.5 \mathrm{~Hz}$. The dominant source of thermal noise for the blade springs arises from thermoelastic dissipation, associated with the thermomechanical properties and geometry of the silicon springs. Therefore, further reductions could be gained through cooling to cryogenic temperature, particularly where the linear coefficient of expansion of silicon is zero $(\sim 18 \mathrm{~K}$ and $\sim 120 \mathrm{~K})$. Moving to a blade material with more beneficial thermomechanical properties at room temperature, such as fused silica, could permit further improvements without the requirement for cryogenics. Further investigations would be required to validate these approaches.

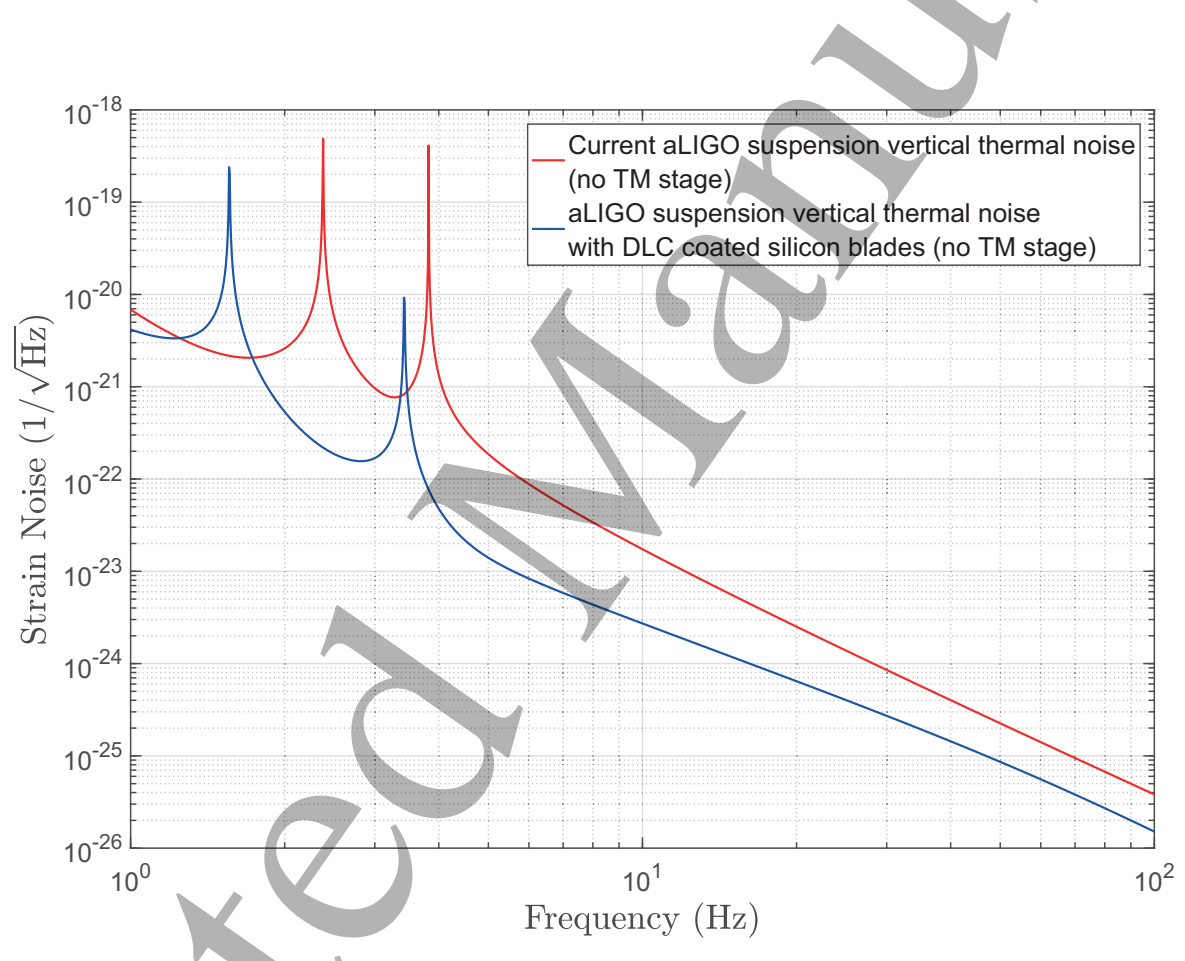

Figure 7: Vertical thermal noise calculated for Advanced LIGO assuming current configuration (red) compared to replacing lower-stage maraging steel blade springs with $1.4 \mathrm{~mm}$ thickness silicon springs coated with $1 \mu \mathrm{m}$ protective DLC layer (blue). The lower monolithic stage (TM stage) is ignored. 
Coatings and surface treatments for enhanced performance suspensions

\section{Conclusions \& further work}

All coatings and surface treatments, with the exception of the magnetron sputtered silica, have improved the tensile and flexural strength of untreated silicon. Base silicon exhibited higher strength than that reported in [26], similar strength to that in [25], and lower than that in [23]. The silicon surface quality is likely to be the dominant factor in determining tensile and flexural strength. Note that the silicon flexures tested in this study were of different geometry to those reported previously [23, 25].

Multilayer DLC coating combined with an extended pre-deposition argon etch step increases the average tensile failure stress of silicon by around $80 \%$ compared to the base material. A number of outlying data points for both single- and multilayer DLC coated silicon show higher tensile strength. Some data points approach the values reported in [23] which utilised samples of smaller cross-section, despite the fact that larger cross-sectional area would be expected to lead to lower tensile strength. This suggests that higher tensile strengths for silicon are achievable with the correct choice of surface treatment.

It appears that the edge polishing slightly degrades the strength of the silicon; this may be explained by the creation of edge defects and/or chips, as shown in [26]. Interestingly, however, this statement does not hold true for multilayer DLC-coated samples, which are stronger on average than the corresponding unpolished samples. A possible explanation for this is that these particular coatings are reasonably thick $(\sim 3 \mu \mathrm{m})$ which, together with the conformal nature of coatings deposited by PECVD, may be enough to fill in any cracks/or chips at the edges, and thus compensate for the increased concentration of defects.

It was shown that substrate orientation within the vacuum chamber during deposition of the DLC films does not have an appreciable effect on the measured tensile strength.

It was shown that in addition to improving the strength of silicon flexures, single- or multilayer DLC coatings also afford an impressive level of protection from abrasion, which may facilitate easier handling/assembly of suspension components and improve their robustness. Similar coatings could be considered for relevant silica and sapphire components, for alternative interferometer configurations.

Replacing the current maraging steel blades of Advanced LIGO with DLCcoated silicon blade springs could offer a 6.4-times reduction in suspension vertical 
thermal noise at $10 \mathrm{~Hz}$, with significant improvement across all frequencies above $3.5 \mathrm{~Hz}$.

Suspension fibres could also be coated with these DLC films, however we note that the thermal noise requirements here would be challenging, due to higher surfaceto-volume ratio and greater influence of the suspension fibres on the thermally driven displacement of the mirrors.

\section{Acknowledgments}

The authors would like to thank the University of the West of Scotland, the University of Glasgow, the University of Strathclyde, the Science and Technology Facilities Council (STFC), the Royal Society, and the Royal Society of Edinburgh for financial support. IWM is supported by a Royal Society Research Fellowship and S Reid supported by a Royal Society Industry Fellowship and Wolfson Merit award. The authors also thank their colleagues within GEO and the LIGO Scientific Collaboration for advice and support. The authors also thank Gerry O'Hare, Liz Porteous, Robert Boyce, Neil Cox, Jim Orr and Colin Craig for assistance in substrate preparation, SEM, fabrication of flexural loading apparatus and substrate mounts. We thank our colleagues in the LSC and VIRGO collaborations and within SUPA for their interest in this work. This paper has LIGO document number LIGO-P1700229.

\section{References}

[1] The LIGO Scientific Collaboration. Advanced LIGO. Class. Quantum Grav., 32(7):074001, 2015.

[2] M Punturo et al. The Einstein Telescope: a third-generation gravitational wave observatory. Classical and Quantum Gravity, 27(19):194002, 2010.

[3] B P Abbott et al. Observation of gravitational waves from a binary black hole merger. Phys. Rev. D, 116:061102, 2016.

[4] J P Sethna, K A Dahmen, and C R Myers. Crackling noise. Nature, 410:242-250, 2010.

[5] G Vajente. Crackling noise in advanced gravitational wave detectors: A model of the steel cantilevers used in the test mass suspensions. Phys. Rev. D, 96:022003, 2017.

[6] X Ni, S Papanikolaou, G Vajente, R X Adhikari, and J R Greer. Probing microplasticity in small-scale fcc crystals via dynamic mechanical analysis. Phys. Rev. Lett., 118:155501, 2017.

[7] A Grill. Diamond-like carbon: state of the art. Diamond Relat. Mater., 8:428-434, 1999.

[8] A Grill. Electrical and optical properties of diamond-like carbon. Thin Solid Films, 355356:189-193, 1999.

[9] J Robertson. Diamond-like amorphous carbon. Mat. Sci. Eng. R, 37:129-281, 2002. 
Coatings and surface treatments for enhanced performance suspensions

[10] S. Reuter, B. Weikamp, R. Büscher, A. Fischer, B. Barden, F. Löer, and V. Bucka. Correlation of structural properties of commercial DLC-coatings to their tribological performance in biomedical applications. Wear, 261:419-425, 2006.

[11] Fraunhofer IST. Name index of carbon coatings, 2012.

[12] K Niranjan Reddy. Double side coating of dlc on silicon by RF-PECVD for AR applicátion. Procedia Engineering, 97:1416-1421, 2014.

[13] F Cemin, C D Boeira, and C A Figueroa. On the understanding of the silicon-containing adhesion interlayer in DLC deposited on steel. Tribol. Int., 94:463-469, 2016.

[14] L F Bonetti, G Capote, L Vieira, E J Corat, and V Trava-Airoldi. Adhesion studies of diamond-like carbon films deposited on $\mathrm{Ti}_{6} \mathrm{Al}_{4} \mathrm{~V}$ substrate with a silicon interlayer. Thin Solid Films, 515:375-379, 2005.

[15] R Birney. Design, Deposition and Characterisation of Protective Modified Multilayered Diamond-Like Carbon Thin Films. PhD thesis, University of the West of Scotland, 2012.

[16] D Lusk et al. Thick DLC films deposited by PECVD on the internal surface of cylindrical substrates. Diamond Relat. Mater., 17:1613-1621, 2008.

[17] R Sharma, P K Barhai, and N Kumari. Corrosion resistant behaviour of DLC films. Thin Solid Films, 516:5397-5403, 2008.

[18] M Azzi, M Paquette, J A Szpunar, J E Klemberg-Sapieha,/and L Martinu. Tribocorrosion behaviour of DLC-coated 316L stainless steel. Wear, 267:860-866, 2009.

[19] C Donnet and A Grill. Friction control of diamond-like carbon coatings. Surf. Coat. Technol., 94-95:456-462, 1997.

[20] L Carbone et al. Sensors and actuators for the advanced LIGO mirror suspensions. Class. Quantum Grav., 29:115005, 2012.

[21] X.L Peng and T.W. Clyne. Mechanical stability of DLC films on metallic substrates Part II Interfacial toughness, debonding and blistering. Thin Solid Films, 312:219-227, 1998.

[22] B P Abbott et al. LIGO: The laser interferometer gravitational-wave observatory. Rep. Prog. Phys., 72:076901, 2009,

[23] G L Pearson, W T Read Jr., and W L Feldman. Deformation and fracture of small silicon crystals. Acta Metall., 5:181-191, 1957.

[24] K E Petersen. Silicon as a mechanical material. Proc. IEEE, 70:42, 1982.

[25] W D Sylwestrowicz. Mechanical properties of single crystals of silicon. Phil. Mag., 7:1825$1845,1962$.

[26] A V Cumming, L Cunningham, G D Hammond, K Haughian, J Hough, S Kroker, I W Martin, R Nawrodt, S Rowan, C Schwarz, and A A van Veggel. Silicon mirror suspensions for gravitational wave detectors. Classical and Quantum Gravity, 31(2):025017, 2014.

[27] A A Griffiths. The phenomena of rupture and flow in solids. Phil. Trans. R. Soc. A, 221:163198, 1921.

[28] S M Hu. Critical stress in silicon brittle fracture, and the effect of ion implantation and other surface treatments. J. Appl. Phys., 53:3576, 1982.

[29] R Jones. The design of the IGR MKII strength testing machine. LIGO Document T1000345, 2010.

[30] ASTM. Standard Test Method for Flexural Strength of Advanced Ceramics at Ambient 
Temperature. C1161-02c Standard, 2008.

[31] ASTM. Standard Test Method for Flexural Properties of Unreinforced and Reinforced Plastics and Electrical Insulating Materials by Four-Point Bending. D-6272-10 Standard, 2010.

[32] C. T. Bodur. Biaxial strength testing of ceramics using square and rectangular specimens. Aust. Ceram. Soc., 50.

[33] C T Chong. Mechanical characterization in failure strength of silicon dice. IEEE Proc. Intersoc. Conf. Therm. Phenom., pages 203-210, 2004.

[34] RSRE. Technical Specification for Infra-Red Optical Coatings. TS1888 standård, 1979.

[35] B J Jones and J J Ojeda. Substrate and material transfer effects on the surface chemistry and texture of diamond-like carbon deposited by plasma enhanced CVD. Surf. Interface Anal., 44:1187-1192, 2012.

[36] B J Jones, S Wright, R C Barklie, J Tyas, J Franks, and A J Reynolds. Nanostructure and paramagnetic centres in diamond-like carbon: effect of Ar dilution in PECVD process. Diamond Relat. Mater., 17:1629, 2008.

[37] C. Zener. Internal friction in solids. II. General theory of thermoelastic internal friction. Phys. Rev., 53:90-99, 1938.

[38] P R Saulson. Thermal noise in mechanical experiments. Phys. Rev. D, 42:2437, 1990.

[39] M. E. Husman, C. I. Torrie, M. V. Plissi, N. A. Robertson, K. A. Strain, and J. Hough. Modeling of multistage pendulums: Triple pendulum suspension for geo 600. Review of Scientific Instruments, 71(6):2546-2551, 2000.

[40] S Reid, G Cagnoli, D R M Crooks, J Hough, P Murray, S Rowan, M M Fejer, R Route, and S Zappe. Mechanical dissipation in silicon flexures. Physics Letters A, 351:205-211, 2006.

[41] I Martin et al. Measurements of a low-temperature mechanical dissipation peak in a single layer of $\mathrm{Ta}_{2} \mathrm{O}_{5}$ doped with $\mathrm{TiO}_{2}$. Class. Quantum Grav., 25:055005, 2008.

[42] GWINC wiki page (https://awiki.ligo-wa.caltech.edu/aLIGO/GWINC) accessed 27nd Jun 2017.

[43] G D Hammond, A V Cumming, J Hough, R Kumar, K Tokmakov, S Reid, and S Rowan. Reducing the suspension thermal noise of advanced gravitational wave detectors. Class. Quantum Grav., 29:124009, 2012.

[44] G Hammond, S Hild, and M Pitkin. Advanced technologies for future ground-based, laserinterferometric gravitational wave detectors. Journal of Modern Optics, 61:S10-S45, 2014.

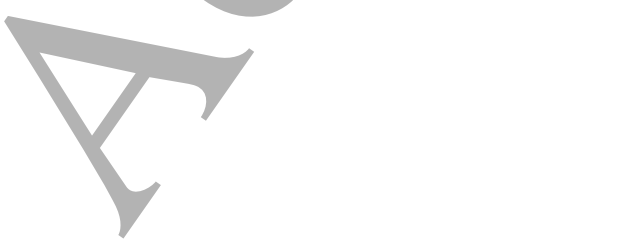

